\title{
Raquialgias na criança
}

Paulo Reis, ${ }^{*}$ Jorge Mineiro**

\section{RESUMO}

As raquialgias são, na criança e no adolescente, menos frequentes do que no adulto, mas traduzem, com maior frequência, a existência de patologia subjacente. Longe de constituírem um gasto excessivo de tempo do clínico, a colheita minuciosa da história clínica e um exame objectivo cuidadoso e sistemático são, indubitavelmente, um dos melhores investimentos do médico que vê crianças com este tipo de sintoma. Permitem abordar correctamente o doente e a sua doença e a solicitação dos exames complementares de diagnóstico mais adequados, em face de uma hipótese diagnóstica correctamente equacionada.

Perante uma criança com raquialgias, a atitude depende da idade, da gravidade dos sinais encontrados na observação e da existência de complicações neurológicas ou outros sinais extra-raquidianos; na sua ausência, é prudente seguir a evolução das queixas durante algum tempo, antes de submeter o doente a uma investigação frequentemente onerosa, consumidora de tempo e com baixa probabilidade de êxito.

Palavras-chave: Criança; Adolescente; Raquialgia.

\section{INTRODUÇÃO}

A s raquialgias são uma das queixas músculo-esqueléticas mais frequentes na população geral adulta, ${ }^{1}$ mas a sua importância é controversa nas crianças e nos jovens. Têm vindo a ser publicados trabalhos cujos resultados contrariam o conceito, comummente aceite, de que as raquialgias são pouco frequentes neste grupo etário..$^{2-4} \mathrm{Num}$ recente trabalho, cerca de $30 \%$ de uma coorte de 1.242 adolescentes entre os 11 e os 17 anos, seguidos prospectivamente durante 4 anos, referia antecedentes de raquialgia, com incapacidade significativa em $30 \%$ dos afectados, o que torna esta situação um problema de saúde pública a considerar. ${ }^{5}$ Outro aspecto que distingue a abordagem deste problema na criança relaciona-se com a etiologia dos sintomas: no adulto, são, sobretudo, os processos degenerativos e os fenómenos psicológicos que subjazem as queixas, enquanto que, na criança, são frequentes as causas orgânicas..$^{6-8}$ Estas diferenças justificam, perante uma criança com raquialgia persistente, uma cuidadosa investigação diagnóstica. ${ }^{7,9}$ Revê-se, neste artigo, a metodologia de avaliação diagnóstica das raquialgias na criança e no adolescente, abordando alguns aspectos específicos da anamnese e do exame objectivo (EO), os exames complemen-

\footnotetext{
*Especialista de Reumatologia em profissão liberal

**Especialista de Ortopedia e de Cirurgia da Coluna Vertebral do Hospital CUF Descobertas, Coordenador da Unidade de Ortopedia e Traumatologia, Director Clínico do Hospital Cuf Descobertas, Consultor de Cirurgia da Coluna Pediátrica do Hospital $D^{a}$ Estefânia, Professor da FML
}

tares de diagnóstico (ECD), as etiologias mais comuns e discutem-se alguns aspectos do diagnóstico diferencial e da terapêutica.

\section{ANAMNESE E EXAME OBJECTIVO}

\section{História Clínica}

A colheita da história clínica e a realização de um EO cuidadoso são passos essenciais na avaliação de uma criança que aparenta estar doente. Se se tratar de uma criança muito jovem, a colheita da anamnese tem de ser feita a partir dos elementos fornecidos pelos pais ou adultos que a acompanham, sendo regra obter-se informações pouco precisas, tais como: irritabilidade, recusa em se sentar ou deambular ou o aparecimento de uma modificação da postura. Nas crianças mais velhas e nos adolescentes, é frequente conseguir-se obter uma melhor caracterização das queixas. Apesar dessas limitações, deve-se, num como noutro grupo, tentar caracterizar as queixas em relação a:
a) modo de início;
b) localização precisa;
c) natureza, intensidade e ritmo da dor;
d) irradiação da dor;
e) duração do quadro clínico;
f) factores desencadeantes do início;
g) manifestações extra-raquidianas coexistentes;
h) nível normal de actividade até ao início das queixas e modificação do mesmo com o início da sintoma- tologia;


i) despiste de história traumática prévia ao início das queixas, caracterizando o tipo e intensidade da lesão e sua relação com os sintomas;

j) coexistência de manifestações sistémicas (febre, perda de peso e anorexia, por exemplo);

k) factores de alívio identificados;

l) sintomas de envolvimento neurológico (fraqueza muscular, alterações da sensibilidade, disfunção dos esfíncteres vesical ou intestinal) e

m) estado geral de saúde e de desenvolvimento psicomotor $^{1,2,10-12}$

\section{Exame Objectivo}

O EO deve incluir uma avaliação geral completa e incidir sobre todo o aparelho músculo-esquelético (não se limitando apenas ao ráquis); o exame neurológico é, igualmente, fundamental. ${ }^{1,2,10}$ Deve observar-se de todos os ângulos a criança ou o adolescente, em ortostatismo (com os membros em extensão e os pés unidos), sentada e durante a mobilização do ráquis, registando-se o alinhamento vertebral, as mobilidades e a existência de espasmo da musculatura paravertebral. ${ }^{10} \mathrm{Com}$ pleta-se o EO com a observação da pele da linha média da face posterior do corpo, já que a existência de alterações a esse nível pode ser um elemento indicador da presença de anomalias estruturais congénitas do ráquis. ${ }^{1}$ A identificação de pontos dolorosos pode orientar a investigação complementar e pode denunciar, por exemplo, o envolvimento das articulações sacro-ilíacas. A exclusão de outras patologias (extra-raquidianas), que podem ter expressão clínica indistinguível, deve ser sempre feita, nomeadamente em relação ao aparelho urinário, tubo digestivo e ao aparelho hepato-bilio-pancreático. Embora menos comum como causa de raquialgias, a patologia ginecológica pode estar implicada, sobretudo nas adolescentes pré-menarca. ${ }^{1,17,18}$ Também algumas infecções sistémicas (brucelose, febre Q, gripe, encefalite, por exemplo) podem apresentar-se com raquialgias. ${ }^{1}$ Devem, avaliar-se, também, a morfologia, dimensões e mobilidades dos membros superiores e inferiores: a existência de deformidades do pé, por exemplo, pode reflectir uma lesão neurológica progressiva. Numa criança muito jovem, a avaliação das mobilidades pode fazer-se observando a criança a realizar actividades normais da sua vida, como, por exemplo a mudança da posição de cócoras

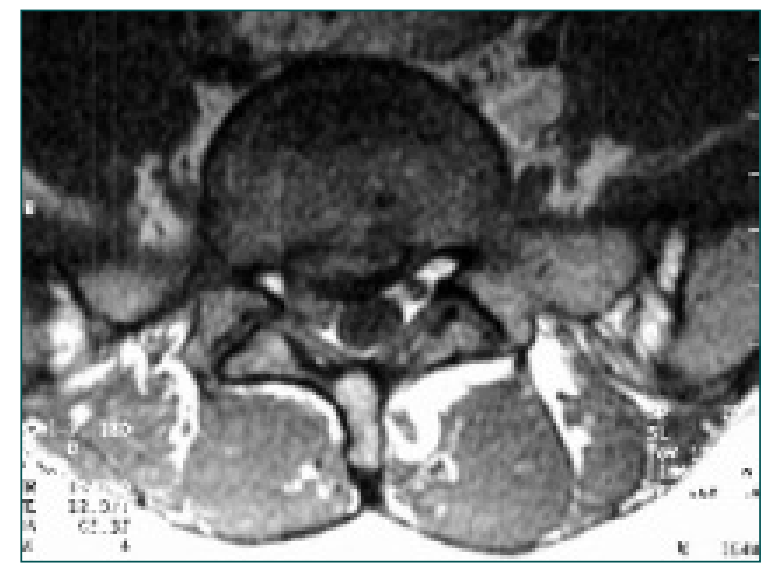

Figura 1. RMN de hérnia discal L5S1 direita num rapaz de 13 anos

até ao ortostatismo. Perante uma criança com lombalgias, deve pesquisar-se sempre o sinal de Laségue, mas é necessário distinguir entre a dor associada à limitação da flexão da coxa sobre o tronco (resultante de uma contractura dos músculos isquio-tibiais), da dor com origem no ráquis, que irradia pelo membro inferior até ao pé (resultante de uma compressão nervosa, por exemplo, por uma hérnia discal) (Figural). ${ }^{2}$

O exame neurológico completo constitui, também, um elemento obrigatório.

\section{Atitude clínica e diagnóstica}

Perante este sintoma, não podem definir-se atitudes nem abordagens diagnósticas padronizadas, devendo orientar-se as investigações de acordo com os dados obtidos na anamnese e EO.

No entanto, podem, genericamente, distinguir-se dois tipos de situações padrão, que condicionam atitudes diferentes:

a) a de uma criança com raquialgias flutuantes e de curta duração (1 a 2 semanas), sem outras manifestações ou incapacidade associadas e

b) um quadro de raquialgias persistentes (mais de 4 semanas), em crianças jovens, condicionando incapacidade para as actividades habituais (desporto, brincar, frequência da escola) e/ou que se acompanham de repercussões sistémicas ou de sinais neurológicos. ${ }^{19}$

No primeiro grupo, aquele em que as queixas resolvem espontaneamente, incluem-se alguns doentes cuja dor tem um componente psicogénico. ${ }^{11,20}$ 
QUADRO I. Sinais de alerta no contexto de raquialgias na criança

Recusa para deambulação*

Dor vertebral persistente

Claudicação

Dor agravada pela actividade física

Dor com componente nocturno marcado

Sinais neurológicos de novo

*Facilmente confundido com quadro de artrite séptica da anca

Globalmente, pode considerar-se que, perante uma criança que apresente sintomatologia dolorosa referida ao aparelho locomotor, a ausência de sinais objectivos de dor ou de limitação da mobilidade sugere que as queixas se devem a um problema pouco grave e que é provável a sua resolução espontânea, ${ }^{20}$ justificando, assim, a atitude expectante advogada por alguns autores.

Por outro lado, existem alguns «sinais de alerta», que obrigam a uma abordagem cuidadosa; o Quadro I resume as manifestações que devem implicar uma atitude mais célere e cuidadosa.

\section{Meios Complementares de Diagnóstico}

A realização de testes laboratoriais ou outros ECD está indicada sempre que exista algum dos «sinais de alarme» (Quadro I)) ou que as queixas sejam crónicas. ${ }^{2}$

\section{Métodos de Imagem}

A avaliação por métodos de imagem deve começar pela realização de estudo por radiologia convencional de toda a coluna, em posição ortostática, em projecções antero-posterior e de perfil. Esta avaliação deve ser complementada pela realização de radiografias em incidências oblíquas direita e esquerda do segmento afectado e, no caso de envolvimento da coluna cervical e lombar, pela realização de radiografias dinâmicas de perfil, em flexão e extensão, para despiste de uma eventual instabilidade segmentar. ${ }^{10}$ Este tipo de abordagem pode permitir obter o diagnóstico em cerca de $60 \%$ dos casos. ${ }^{21}$ Algumas patologias específicas, como as deformidades, requerem a realização de radiografias em posições específicas: perfil em ortostatismo e em decúbito dorsal para avaliar a flexibilidade de uma cifose, ou as radiografias em antero-posterior com flexão lateral máxima direita e esquerda, na avaliação de uma escoliose. $^{1}$

Perante a suspeita de uma lesão menos frequente, como uma hérnia discal ou um tumor, é necessária a realização de exames mais sofisticados, como a tomografia axial computorizada (TAC), a ressonância magnética (RMN) ou a cintigrafia óssea. Nestas circunstâncias, a atitude mais correcta será referenciar prontamente a criança para um serviço de Ortopedia Pediátrica.

\section{Investigação laboratorial}

Na maioria das situações, não é necessário realizar testes laboratoriais para avaliar uma criança com raquialgias. ${ }^{2,10}$ Existem, contudo, alguns casos, como a suspeita de um processo infeccioso ou inflamatório, em que se justifica o pedido da velocidade de sedimentação (VS), proteína $C$ reactiva (PCR) e de um hemograma.

\section{Etiologias específicas e diagnóstico diferencial}

Existem múltiplas classificações das causas de raquialgias na criança e no adolescente; escolhemos, aqui, a classificação proposta por Thompson ${ }^{10}$ (Quadro II), que resume bem as diversas etiologias.

A doença de Scheuermann constitui, provavelmente, a causa mais comum de raquialgias das regiões torácica e toraco-lombar, enquanto a espondilólise ou a espondilolistese são as causas mais comuns das raquialgias lombares e lombo-sagradas. No entanto, perante uma criança ou adolescente com raquialgias, o médico deve, por uma questão de rigor metodológico, equacionar as diferentes categorias diagnósticas. Em seguida procede-se a uma breve discussão de algumas entidades nosológicas pertencentes aos pincipais grupos de causas, referidos no Quadro II.

\section{Patologia do desenvolvimento}

Neste grupo incluem-se a escoliose idiopática e a doença de Scheuermann.

A escoliose idiopática é a forma mais comum de escoliose na criança e é, em regra, uma condição assintomática, pelo que a existência de sintomas dolorosos deve levantar a hipótese da coexistência de outras patologias, como uma infecção ou tumor. Consoante a idade em que surge a deformidade da coluna, podemos 


\begin{tabular}{|c|c|}
\hline \multicolumn{2}{|c|}{$\begin{array}{l}\text { QUADRO II. Diagnóstico diferencial das raquialgias na } \\
\text { criança }\end{array}$} \\
\hline Congénita & $\begin{array}{l}\text { Diastematomielia } \\
\text { Anomalias da coluna cervical }\end{array}$ \\
\hline Desenvolvimento & $\begin{array}{l}\text { Escoliose dolorosa } \\
\text { Cifose do adolescente (D. Scheuermann) }\end{array}$ \\
\hline Traumática & $\begin{array}{l}\text { Fractura oculta } \\
\text { Contractura muscular } \\
\text { Espondilolíse ou espondilolistese } \\
\text { Hérnia discal } \\
\text { Instabilidade cervical alta } \\
\text { «Slipped vertebral apophysis» }\end{array}$ \\
\hline Infecciosa & $\begin{array}{l}\text { Discite } \\
\text { Osteomielite vertebral } \\
\text { Tuberculose }\end{array}$ \\
\hline Sistémica & $\begin{array}{l}\text { Infecções crónicas } \\
\text { Doenças do armazenamento } \\
\text { Osteoporose Juvenil }\end{array}$ \\
\hline Artrite Juvenil & $\begin{array}{l}\text { Artrite Reumatóide } \\
\text { Espondilite anquilosante }\end{array}$ \\
\hline $\begin{array}{l}\text { Neoplásica } \\
\text { (benigno } \\
\text { e maligno) }\end{array}$ & $\begin{array}{l}\text { Benigno } \\
\text { - Osteoma Osteoide } \\
\text { - Osteoblastoma } \\
\text { - Quisto Ósseo Aneurismático } \\
\text { - Granuloma Eosinófilo } \\
\text { Maligno } \\
\text { - Sarcoma Ewing } \\
\text { - Osteocarcoma } \\
\text { - Metastático }\end{array}$ \\
\hline Psicogénica & \\
\hline
\end{tabular}

considerar três tipos de escoliose idiopática:

a) infantil, quando surge até aos 3 anos de idade;

b) juvenil, se ocorre dos 3 anos até à puberdade;

c) do adolescente, quando surge durante ou após a puberdade.

A topografia das curvaturas é, também, importante e varia consoante a idade de início da escoliose. Nas escolioses idiopáticas infantis (que, em $85 \%$ dos casos, resolvem espontaneamente) são mais frequentes as curvaturas toraco-lombares esquerdas, enquanto que nas escolioses idiopáticas juvenil e do adolescente são as curvaturas torácicas direitas ou duplas curvaturas (torácica direita e lombar esquerda). Refira-se que apenas 1,6\% de uma série de 1.662 doentes adolescentes tinha curvatura esquerda e destes, $33 \%$ tinham como causa lesões neurológicas, tumores e doenças neuromusculares, entre outras. ${ }^{30}$

Perante uma escoliose dolorosa, pelo facto de nas escolioses idiopáticas a semiologia ser pouco rica, recomenda-se a realização de uma cuidadosa avaliação músculo-esquelética e neurológica. ${ }^{2,10,26} \mathrm{~A}$ avaliação imagiológica depende dos sintomas referidos e, logicamente, da suspeita diagnóstica que estes suscitem. Para além da radiologia convencional em incidências extralongas de pé (em dois planos perpendiculares AP e perfil), deverá pedir-se uma cintigrafia óssea e estudo com TAC e RMN, sempre que exista suspeita de outra patologia. No entanto, a hipótese de outro diagnóstico deve ser discutida com o centro de referenciação de Ortopedia Pediátrica, no sentido de evitar a realização de ECD desnecessários.

A doença de Scheuermann é a causa mais comum de cifose estrutural da coluna torácica e toraco-lombar em adolescentes, afectando 20 a $30 \%$ da população geral e parecendo apresentar uma prevalência major em atletas (halterofilismo, futebol, ginástica). ${ }^{31-35}$ Corresponde a uma cifose torácica, associada a uma deformação cuneiforme dos corpos vertebrais e a irregularidades dos pratos vertebrais; o diagnóstico baseia-se nos achados em radiograma de perfil do ráquis e os critérios de diagnóstico são os propostos por Sorenson. ${ }^{36}$ Este autor definiu a necessidade de envolvimento de, pelo menos, 3 vértebras adjacentes, com uma angulação de $75^{\circ} \mathrm{em}$ cada vértebra, para o estabelecimento do diagnóstico. Parece apresentar um importante componente genético, mas mantém-se uma doença de etiologia desconhecida, embora recentemente tenha sido identificada uma deficiente síntese das fibras de colagénio e da matriz intersticial a nível dos pratos vertebrais, contribuindo para o seu enfraquecimento. Esta menor resistência favorece o aparecimento de herniações discais intra-ósseas nos corpos vertebrais, condicionando colapso discal e lesão da parte anterior do corpo da vértebra, que provoca uma anomalia do crescimento da vértebra, com a consequente deformação. Afecta, tipicamente, adolescentes do sexo masculino, com uma cifose torácica progressiva; a dor é frequente, localizando-se, sobretudo, no apex da cifose (geralmente entre a sétima e a oitava vértebra dorsal, mas podendo localizar-se na coluna lombar, se existir uma cifose muito 
grave). As queixas podem agravar-se pelo ortostatismo, pela posição sentada e pela actividade física e tendem a desaparecer com o fim do crescimento, a menos que as deformidades residuais sejam muito importantes. Não há, habitualmente, défices neurológicos associados; no entanto, as forças anormais que se exercem na coluna lombar inferior resultam num aumento do risco de desenvolvimento de espondilólise, sobretudo a nível da quinta vértebra lombar. ${ }^{37,38} \mathrm{O}$ tratamento da doença de Scheuermann baseia-se na gravidade da deformação, presença de dor e idade do doente e pode passar apenas pela vigilância, pelo recurso a um colete tipo Milwaukee ou, numa minoria de doentes, pela cirurgia.

\section{Patologia traumática}

É comum a referência a um traumatismo antecedendo o início das raquialgias, mas não deve aceitar-se imediatamente a origem traumática dos sintomas: ${ }^{7}$ é fundamental caracterizar a natureza e intensidade do mecanismo de lesão proposto e a sua relação temporal com o início das queixas. Sabe-se que, em $30 \%$ das crianças e adolescentes saudáveis surgem raquialgias após o aumento recente da actividade física ou início de um desporto fisicamente exigente. ${ }^{13}$ No entanto, as raquialgias recorrentes e/ou persistentes, em crianças e adolescentes que fazem muito desporto, só podem ser consideradas síndromes de hiperutilização ou lesões musculares depois de se ter excluída a possibilidade de patologia grave, já que este tipo de evolução pode ser a primeira manifestação de uma espondilólise juvenil. ${ }^{1}$ Foi demonstrada a associação entre o desenvolvimento de alterações degenerativas precoces na coluna lombar e a ocorrência de lesões durante o surto de crescimento na adolescência. ${ }^{16}$ Este facto justifica a necessidade de caracterizar adequadamente a actividade física presente e passada da criança ou adolescente, bem como o registo de eventuais traumatismos, como parte da avaliação perante queixas de raquialgias.

Neste grupo incluem-se as fracturas ocultas, as lesões musculares, a espondilólise e a espondiolistese, as hérnias discais e o «arrancamento» do rebordo dos pratos vertebrais em crescimento («slipped vertebral apophysis»).

As fracturas ocultas ocorrem, tipicamente, após traumatismos de baixa energia (de que são exemplo as que- das durante actividades desportivas), mas podem verificar-se na sequência de traumatismos mais graves, não sendo reconhecidas. As localizações preferenciais são as apófises transversas e espinhosas e as apófises articulares, sendo, muitas vezes, pouco visíveis nos estudos por radiologia convencional. Ocasionalmente, este tipo de lesão pode ocorrer noutras localizações, como por exemplo, o sacro. ${ }^{39}$ Se uma criança refere queixas persistentes após um traumatismo e a radiologia convencional é inconclusiva, está indicada a realização de uma cintigrafia óssea: a localização sugerida pela hiperfixação na cintigrafia será, depois, estudada por TAC. O tratamento é geralmente conservador: restrição da actividade física até ao desaparecimento dos sintomas e um programa de fisioterapia adequado. É fundamental um diagnóstico preciso da localização da fractura, já que, embora a maior parte cure espontaneamente, as que interessam os pedículos podem evoluir para a instabilidade. ${ }^{10}$

As lesões musculares são comuns e diagnosticam-se a partir da história do início das queixas e da observação, aliadas a um estudo por radiologia normal. Este tipo de lesão é, geralmente, produzido por traumatismo directo ou resulta de uma hiperutilização (por actividade desportiva, por exemplo). O tratamento faz-se com repouso, seguido pela retoma das actividades desportivas sob uma correcta orientação e com técnicas de treino adequadas. ${ }^{10}$

A espondilólise e a espondilolistese constituem lesões comummente envolvidas na génese de raquialgias na criança ${ }^{37,40-42}$ Atingem cerca de 4 a $6 \%$ da população gera $1,{ }^{1}$ mas existem grandes variações com a raça e o sexo, ${ }^{43}$ sendo possível a ocorrência de um padrão familiar. ${ }^{32} \mathrm{~A}$ área mais frequentemente envolvida é a transição entre a $5^{\mathrm{a}}$ lombar e a $1^{\mathrm{a}}$ sagrada. ${ }^{10}$ São mais comuns após os 7 anos de idade, sendo maior a prevalência durante o surto de crescimento da adolescência. ${ }^{1}$ Habitualmente, são raquialgias de baixa intensidade, localizadas na região lombar, atingindo, por vezes, as regiões glúteas e as faces posteriores das coxas. Há agravamento com o exercício e alivío com o repouso e diminuição da carga de esforço, sendo raras queixas ou alterações no exame neurológico. A observação evidencia, frequentemente, uma contractura dos músculos isquio-tibiais, que se traduz numa marcha típica, com a bacia basculada para trás, flexão limitada das coxo-fe- 

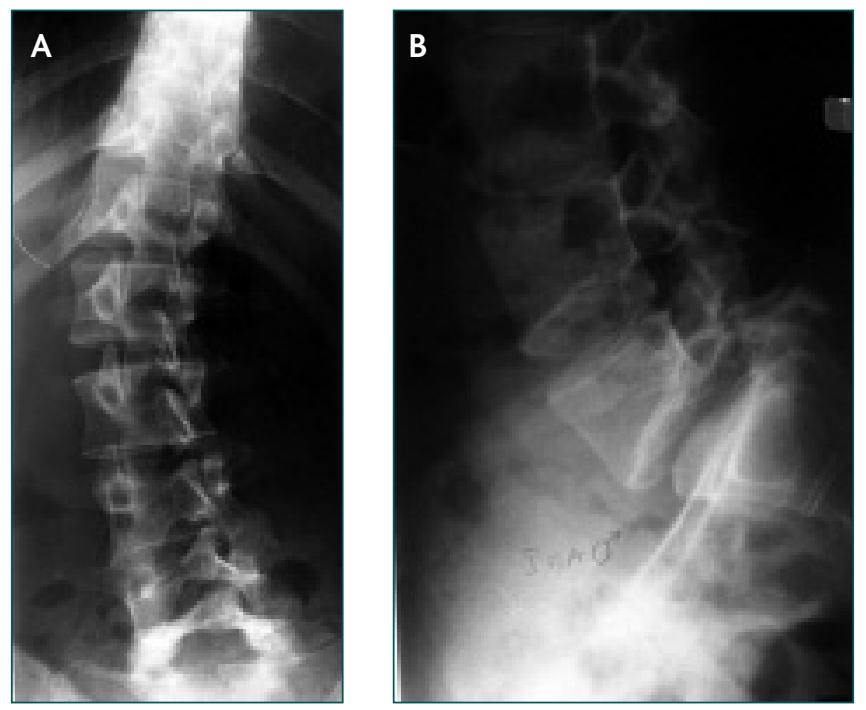

Figura 2. A. Escoliose secundária a espondilolistese de L5S1 B. Espondilolistese L5S1 por defeito do pars interarticularis de L5

murais, e uma amplitude de passo muito diminuída; pode, também, identificar-se uma lordose lombar aumentada e dor à palpação das apófises espinhosas e dos músculos para-vertebrais. Em 25 a 50\% dos casos, este tipo de lesão associa-se a uma escoliose, ${ }^{44}$ sendo, também, comum encontrar uma espinha bífida oculta associada ${ }^{40} \mathrm{O}$ diagnóstico faz-se, habitualmente, com base nos achados em radiologia convencional, em projecção antero-posterior e de perfil em ortostatismo (sendo esta última particularmente útil), mas a realização de projecções oblíquas possibilita uma melhor identificação do defeito do «pars interarticularis» (Figura 2). O tratamento (conservador ou cirúrgico) da espondilolistese depende, essencialmente, do grau inicial de deslizamento e da progressão desse mesmo deslizamento.

As hérnias discais podem ocorrer, raramente, nas crianças e nos adolescentes, constituindo apenas $0,4 \mathrm{a}$ $5,9 \%$ de todos os doentes com hérnia discal ${ }^{46,49}$ e 2 a $3 \%$ de todas as intervenções cirúrgicas por esta patologia. ${ }^{1,2,10,11} \mathrm{~A}$ sua raridade não deve levar à sua exclusão como causa de raquialgia neste grupo etário. Embora a maioria dos casos surja entre os 12 e os 16 anos, também podem ocorrer em crianças mais jovens. ${ }^{50,51}$ A localização mais frequente das hérnias é nos discos entre L4 e L5 e entre L5 e S1. As manifestações clínicas são semelhantes às do adulto mas, em mais de
$50 \%$ dos jovens, existe referência a um traumatismo antecedendo o início do quadro. As queixas mais comuns são lombo-ciatalgias ( $2 / 3$ das crianças) e claudicação da marcha (em $1 / 3$ dos casos) ${ }^{26}$ Tal como no adulto, a dor agrava com o ortostatismo e alivia com o repouso, podendo, também, agravar-se com as manobras de Valsalva e encontrar-se um sinal de Lasègue positivo. Por vezes, a queixa inicial é a incapacidade para a marcha, por impossibilidade de realizar a extensão do joelho, condicionando uma deformação em flexão na anca homolateral, o que pode provocar uma centralização do clínico no membro inferior e, desse modo, atrasar o diagnóstico. ${ }^{1}$ Outro tipo de apresentação clínica, que surge em $20 \%$ dos casos, é uma escoliose dolorosa. As alterações da postura e da marcha são mais comuns do que as alterações sensório-motoras, mas cerca de 30 a $40 \%$ destes doentes apresentarão diminuição da força. ${ }^{1} \mathrm{O}$ diagnóstico faz-se pela demonstração da hérnia por $\mathrm{TAC}$, radiculografia ou $\mathrm{RMN},{ }^{11}$ mas esta última parece ser a que apresenta maior sensibilidade diagnóstica. A terapêutica é semelhante a do adulto, mas existe uma maior probabilidade de não haver resposta com a terapêutica médica. ${ }^{1}$ A restrição de actividade, o repouso no leito e a prescrição de analgésicos devem constituir a abordagem inicial, colocando-se a indicação cirúrgica se houver falência destas medidas.

\section{Patologia inflamatória}

Neste grupo incluem-se a artrite crónica juvenil e as espondiloartropatias.

A artrite crónica juvenil pode ter, como forma de apresentação, uma cervicalgia, mas a localização exclusiva da doença à coluna cervical é uma raridade, pelo que uma história clínica e observação correctas revelam o envolvimento de outras áreas articulares. A limitação da mobilidade cervical pode preceder o aparecimento de alterações radiológicas. A avaliação laboratorial poderá ter um papel auxiliar no diagnóstico. $\mathrm{O}$ tratamento deve ser orientado por um reumatologista com experiência no seguimento de crianças, mas o facto de existir uma incidência aumentada de escoliose justifica um apoio contínuo do ortopedista. ${ }^{53}$

As espondiloartropatias, e, mais específicamente, a espondilartrite anquilosante, podem surgir, também, na criança. Em 8 a 9\% dos casos, a doença tem os seus 
primeiros sintomas entre os 10 e os 15 anos, havendo um atraso no estabelecimento do diagnóstico. Existe, frequentemente, uma história familiar de psoríase, doença inflamatória do intestino ou episclerite, sendo estes dados relevantes. O EO apresenta, tipicamente, uma diminuição da expansão e mobilidade torácicas. No início do curso da doença, a avaliação por radiologia convencional é, geralmente, normal, mas pode haver hiperfixação nas sacro-ilíacas na cintigrafia óssea. $\mathrm{O}$ tratamento baseia-se na administração de anti-inflamatórios não esteróides e num correcto programa de fisioterapia, para manutenção da mobilidade.
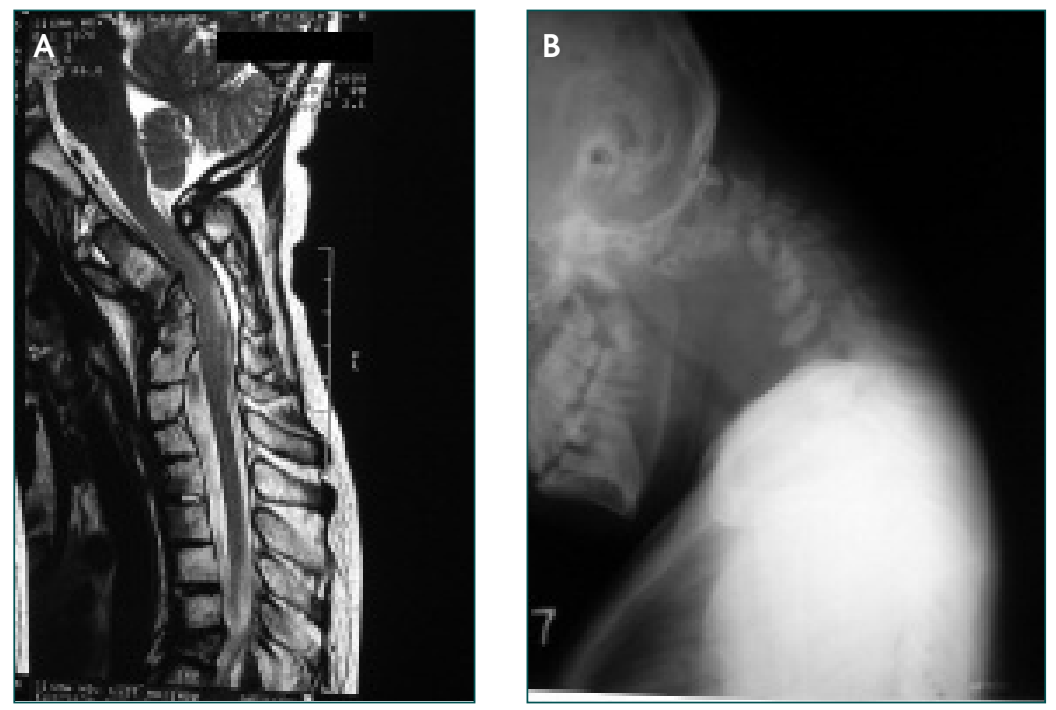

Figura 3. A. Osteomielite da coluna cervical com deformidade cifótica B. RMN cervical mostra a destruição dos corpos vertebrais

\section{Patologia infecciosa}

Neste grupo incluem-se as discites, as osteomielites vertebrais e a tuberculose.

A discite é uma inflamação do disco intervertebral, habitualmente provocada por uma infecção bacteriana, que ocorre com maior frequência nos doentes mais jovens ( 4 a 10 anos) e com uma incidência particular na coluna lombar. Ao contrário do adulto, na criança o disco é vascularizado por vasos que provêm dos pratos vertebrais. A etiologia das infecçöes na criança presume-se, assim, ser a disseminação hematogénica de bactérias com focalização no disco. ${ }^{54} \mathrm{O}$ agente mais frequente é o Staphylococcus aureus, mas apenas em 20 a $50 \%$ das tentativas é possível o isolamento do agente por biópisa com agulha.$^{55}$ As manifestações clínicas dependem da idade da criança: na muito jovem, as discites lombares podem ser de difícil reconhecimento, porque as queixas dolorosas podem ser referidas na anca, perna ou no abdómen. ${ }^{6,32}$ Antes dos 3 anos, a manifestação principal pode ser apenas uma recusa em andar, mas, após essa idade, a clínica pode ser a de uma doença sistémica. $\mathrm{O}$ adolescente referirá, geralmente, queixas mais localizadas de raquialgias, o que facilita e orienta o diagnóstico. Por vezes, é possível recolher uma história de doença febril prévia, com astenia. A observação pode revelar, apenas, uma contractura da musculatura paravertebral ou uma resistência a todas as manobras diagnósticas que impliquem mobilização do raquis. A VS está, geralmente, elevada, mas pode não existir leucocitose. A avaliação por radiologia convencional é, habitualmente, normal no início, já que são necessárias 2 a 4 semanas para que possam surgir os primeiros sinais de estreitamento do disco ou de erosões irregulares dos pratos vertebrais; a esclerose reaccional aparece mais tarde. No entanto, se, no adulto, a regra é o colapso discal por destruição do disco, nos mais jovens assiste-se, por vezes, a um «refazer» da altura do próprio disco, após a resolução do processo. Está indicada a realização de RMN (Figura 3) ou cintigrafia óssea (sensibilidade 80 a 90\%), nas fases precoces de evolução da doença. ${ }^{2}$ Em regra, esta situação evolui para a cura, com resolução completa dos sintomas e, numa fase posterior, com maior ou menor destruição dos discos intervertebrais afectados.

Ao contrário do que se verifica nas discites, a osteomielite vertebral tem um pior prognóstico. Apresenta-se, geralmente, com um quadro séptico mais importante e, em certas circunstâncias, requer, mesmo, uma terapêutica cirúrgica. As imagens por radiologia convencional são muito sugestivas: osteólise, deformação grave e destruição, sendo frequente o colapso vertebral e as deformações residuais do segmento afectado (Figura 4). A terapêutica de eleição desta situação é cirúrgica.

A tuberculose e outros agentes (quisto hidático, por 


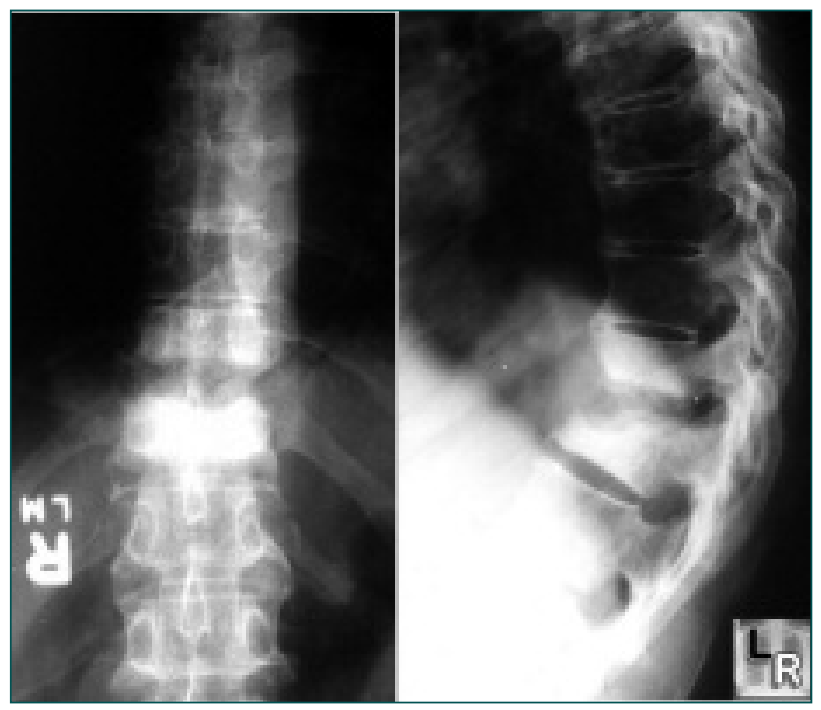

Figura 4. Osteomielite vertebral com destruição dos pratos discais e disco intervertebral

exemplo), ${ }^{56}$ podem constituir, igualmente, causa de infecção no ráquis. A tuberculose envolve típicamente o disco e a vertebra adjacente, mas pode atingir várias vértebras. O processo inicia-se, geralmente, na metade anterior do corpo da vértebra, junto ao disco, sendo este envolvido posteriormente. $\mathrm{O}$ aspecto radiográfico mais comum é a coexistência de um colapso do disco com lesão das 2 vértebras adjacentes. A VS está, habitualmente, elevada e o teste de Mantoux é positivo. O diagnóstico etiológico só se pode estabelecer pelo isolamento do agente ou pela demonstração de histologia típica, sendo necessário realizar uma biópsia por agulha. O tratamento faz-se com imobilização da coluna vertebral com colete de gesso ou outra ortótese, associada a terapêutica antibacilar tripla. Os abcessos de grandes dimensões devem ser drenados após o início da quimioterapia. ${ }^{11}$

\section{Neoplasias}

As neoplasias podem localizar-se, quer nos componentes ósseos, quer nos elementos neurais. As neoplasias ósseas da criança são raras, mas ainda mais raras são as neoplasias de origem em estruturas neurológicas. A sua raridade obriga a manter um alto índice de suspeita para ser possível um diagnóstico precoce. As zonas mais envolvidas são a coluna lombar e torácica. A quei- xa mais frequente é a dor (geralmente persistente e sem ritmo característico), associada, ocasionalmente, a sinais neurológicos, mas a fraqueza muscular, claudicação da marcha ou alterações esfinctéricas podem constituir outras formas de apresentação; a associação de lombalgia e ciatalgia bilateral sugere um tumor intradural. A observação pode evidenciar dor local, espasmo muscular, escoliose ou limitação da mobilidade. A radiologia convencional apresenta, geralmente, alterações: fracturas vertebrais de tipo compressivo, erosões do arco neural ou do corpo vertebral, calcificações paravertebrais e alargamento do canal raquidiano. A realização de TAC, RMN ou outros ECD é, também, necessária para o esclarecimento cabal deste tipo de situação.

\section{Tumores benignos}

Apesar de raras, as neoplasias benignas constituem cerca de $70 \%$ dos tumores primários da coluna vertebral nas crianças. Neste grupo, os tumores mais frequentes são os osteomas osteóides, os osteoblastomas e os quistos ósseos aneurismáticos.

\section{Tumores malignos}

Apesar de muito raros, os tumores malignos da coluna na criança têm, ainda hoje, um prognóstico muito reservado, pela gravidade da lesão e pelas particularidades da estrutura anatómica onde estão inseridos. Os tumores malignos mais frequentes na coluna da criança/adolescente são o sarcoma de Ewing e o osteosarcoma.

\section{OUTRAS PATOLOGIAS}

Podem, ainda, surgir raquialgias em crianças e adolescentes noutras situações, como a fibromialgia, ${ }^{26}$ ou na sequência de cirurgia ortopédica de alongamento de membros inferiores, e noutras, primáriamente raquidianas ou extraraquidianas. Uma correcta metodologia de avaliação permitirá distinguir as situações em que as raquialgias são o problema principal ou, apenas, o reflexo de outra patologia.

\section{CONCLUSÃO}

As raquialgias são, na criança e no adolescente, menos comuns do que no adulto, mas traduzem, com maior frequência, a existência de patologia subjacente. Longe de constituírem um gasto excessivo de tempo do clí- 
nico, a colheita minuciosa da história clínica e um exame objectivo cuidadoso e sistemático, são, indubitavelmente, um dos melhores investimentos do médico que vê crianças com este tipo de sintoma. Permitem a abordagem correcta do doente e da sua doença e a solicitação adequada dos ECD, em face de uma hipótese diagnóstica correctamente equacionada.

Perante uma criança com raquialgias, a atitude depende da idade, da gravidade dos sinais encontrados na observação e da existência de complicações neurológicas ou outros sinais extrarraquidianos; na sua ausência, é prudente seguir a evolução das queixas durante algum tempo, antes de submeter o doente a uma investigação frequentemente onerosa, consumidora de tempo e com baixa probabilidade de êxito.

\section{REFERÊNCIAS BIBLIOGRÁFICAS}

1. White JI, Gardner VO, Takeda H. Back pain in the pediatric patient: assessment and differential diagnosis. Spine: State of the Art Reviews Series 1990, 4 (1): 1-24.

2. Sponseller PD. Back pain in children. Curr Opin Pediatr 1994 Feb; 6 (1): 99-103.

3. Salminen JJ, Maki P, Oksanen A, Pentti J. Spinal mobility and trunk muscle strenght in 15 year old schoolchildren with and without low-back pain. Spine 1992 Apr; 17 (4): 405-11.

4. Salminen JJ, Pentti J, Terho P. Low back pain and disability in 14-yearold schoolchildren. Acta Paediatr 1992 Dec; 81 (12): 1035-9.

5. Olsen TL, Anderson RL, Dearwater SR, Kriska AM, Cauley JA, Aaron DJ, et al. The epidemiology of low back pain in an adolescent population. Am J Public Health 1992 Apr, 82 (4): 606-8.

6. Bunnell WP. Back pain in children. Orthop Clin North Am 1982 Jul; 13 (3): 587-604.

7. Hensinger RN. Back pain in children. In: Bradford DS, Hensinger RN, editors. The Pediatric Spine. New York: Thieme-Stratton; 1985. p. 41 - 60.

8. King HA. Back pain in children. Pediat Clin North America 1984 Oct; 31 (5): 1083-95.

9. Turner PG, Green JH, Galasko CS. Back pain in childhood. Spine 1989 Aug; 14 (8): 812-4.

10. Thompson GH. Back pain in children. J Bone Joint Surg Am 1993 June $1 ; 75$ (6): 928- 38.

11. I. Galasko CS. Back pain in children. In: Jayson Mi, Dixon Al, editors. The lumbar spine and back pain. 4th ed. New York: Churchill Livingstone; 1992. p. 603-17.

12. King HA. Evaluating the child with back pain. Pediat Clin North Am 1986 Dec; 33 (6): 1489-93.

13. Balagué F, Dutoit G, Waldburger M. Low back pain in school children: an epidemiological study. Scan J Rehab Med 1988; 20 (4): 175-9.

14. Fairbank JC, Pynsent PB, Van Poortvliet JA. Influence of anthropometric factors and joint laxity in the incidence of adolescent back pain. Spine 1984 Jul-Aug; 9 (5): 461-4.

15. Stanitski CL. Management of sports injuries in children and adolescents.
Orthop Clin North Am 1988 Oct; 19 (4): 689-98.

16. Kujala UM, Erkintalo MO, Taimela S, Salminen JJ, Kaprio J. Role of acute injury during adolescent growth spurt in development of lumbar spine abnormalities. Lancet 1994 Oct 8; 344 (8928): 1020.

17. Deathe $A B$. Hematometra as a cause of lumbar radiculopathy: a case report. Spine 1993 Oct 1; 18 (13): 1920-1.

18. Harrison CS. Hematocolpos as a cause of low back pain: a case report. Spine 1991 Aug; 16 (8): 985-6.

19. Thompson GH. Back pain in children. J Bone Joint Surg Am 1993 June $1 ; 75$ (6): 928- 38.

20. Staheli LT. Pain of musculoskeletal origin in children. Curr Opin Rheumatol 1992 Oct; 4 (5): 748-52.

21. Turner PG, Green JH, Galasko CS. Back pain in childhood. Spine 1989 Aug; 14 (8): 812-4.

22. Conway JJ. Radionuclide bone scintigraphy in pediatric orthopedics. Pediatr Clin North Am 1986 Dec; 33 (6): 1313-34.

23. Forristall RM, Marsh HO, Pay NT. Magnetic resonance imaging and contrast CT of the lumbar spine. Comparison of diagnostic methods and correlation with surgical findings. Spine 1988 Sep; 13 (9): 1049-54.

24. Packer RJ, Zimmerman RA, Sutton LN, Bilaniuk LT, Bruce DA, Schut L. Magnetic resonance imaging of spinal cord disease of childhood. Pediatrics 1986 Aug; 78 (2): 251-6.

25. Modic M T, Feiglin DH, Pirauno Dw, Boumphrey F, Weinstein MA, Duchesneau PM, et al. Vertebral osteomyelitis: assessment using MR. Radiology 1985 Oct; 157 (1): 157-66.

26. Balagué F, Nordin M. Back pain in children and teenagers. Baillière's Clin Rheumatol 1992 Oct; 6(3): 575-93.

27. Winter RB. Spinal problems in pediatric orthopaedics. In: Monissy RT, editor. Lovell and Winter's Pediatric Orthopaedics. 3rd ed. Philadelphia: J B Lippincott; 1990. p. 6625-702.

28. Ks1amcbi A, Thompson GH. Congenital anomalies of the spine. In: Dee R, Mango E, Hurst LC, editors. Principles of Orthopaedic Practice. New York: McGraw-Hill; 1989. p. 838-60.

29. Rasool MN, Govender S, Naidoo KS, Moodley M. Foot deformities and occult spinal abnormalities in children: a review of 16 cases. J PediatR Orthop 1992 Jan; 12 (1): 94-9.

30. Coonrad RW, Richardson WJ, Oakes WJ. Left thoracic curves can be different. Orthop Trans 1985, 9: 126-7.

31. Commandré FA, Gagnerie G, Zakarian M,Alaoui M, Fourré JM, Bouzayen A. The child, the spine and sport. J Sports Med and Phys Fitness 1988 Mar; 28 (1): 11-9.

32. Afshani E, Kuhn JP. Common causes of low back pain in children. Radiographics 1991 Mar; 11 (2): 269-91.

33. Michieli LJ. Overuse injuries in children's sports: the growth factor. Orthop Clin North Am 1983 Apr; 14 (2): 337-60.

34. Vautravers P, Martin JC, Lecocq J, Zimmermann A. Sports et lombalgies. Journal de Traumatologie du Sport 1990; 7 (2): 90-9.

35. Swärd L, Hellstrom M, Jacobsson B, Peterson L. Back pain and radiologic changes in the thoraco-lumbar spine of athletes. Spine 1990 Feb; 15 (2): 124-9.

36. Sorensen KH. Scheuermann's Juvenile Kyphosis; Clinical appearances, radiography, aetiology and prognosis. Copenhagen: Munksgaard; 1964.

37. Hensinger RN. Spondylolysis and spondylolisthesis in children and adolescents. J Bone and Joint Surg Am 1989 Aug; 71 (7): 1098-107. 
38. Ogilvie JW, Sherman J. Spondylolysis in Scheuermann's disease. Spine 1987 Apr; 12 (3): 251-3.

39. Grier D, Wardell S, Sarwark J, Poznanski AK. Fatigue fractures of the sacrum in children: two case reports and a review of the literature. Skeletal Radiol 1993 Oct; 22 (7): 515-8.

40. Bell DF, Ehrlich MG, Zaleske DJ. Brace treatment for symptomatic spondylolisthesis. Clin Orthop Relat Res 1988 Nov; 236: 192-8.

41. Wiltse LL, Winter RB. Terminology and measurement of spondylolisthesis. J Bone and Joint Surg Am 1983 Jul; 65 (6): 768-72.

42. Ishikawa S, Kumar SJ, Torres BC. Surgical treatment of dysplastic spondylolisthesis: results after in situ fusion. Spine 1994 Aug 1; 19 (15): 1691-6.

43. Crawford AH. Operative treatment of spine fractures in children. Orthop Clin North Am 1990 Apr; 21 (2): 325-39.

44. Burkus JK, Lonstein JE, Winter RB, Denis F. Long-term evaluation of adolescents treated operatively for spondylolisthesis: a comparison of in situ arthrodesis only with in situ arthrodesis and reduction followed by immobilization in a cast. J Bone and Joint Surg Am 1992 Jun; 74 (5): 693-704.

45. Pizzutillo PD, Hummer CD 3rd. Nonoperative treatment for painflul adolescent spondylolysis or spondylolisthesis. J Pediat Orthop 1989 SepOct; 9 (5): 538-40.

46. Nelson CL, Janecki CJ, Gildenberg PL, Sava G. Disk protusions in the young. Clin Orthop Relat Res 1972; 88: 142-50.

47. Gumzburg R, Fraser RD, Fraser GA. Lumbar intervertebral disc prolapse in teenage twins: a case report and review of the litterature. J Bone Joint Surg Br 1990 Sep; 72 (5): 914-6.

48. Yasuma T, Koh S, Okamura T, Yamauchi Y. Histological changes in aging lumbar intervertebral discs: their role and protrusions and prolapses. J Bone Joint Surg Am 1990 Feb; 72 (2) 220-9.
49. Varlotta GP. Brot MD, Kelsey JL, Golden AL. Familial predispositon for herniation of a lumbar disc in patients who are less than twenty-one years old. J Bone Joint Surg Am 1991 Jan; 73: 124-8.

50. Callahan DJ, Pack LL, Bream RC, Hensinger RN. Intervertebral disc impingement syndrome in a child. Report of a case and suggested pathology. Spine 1986 May; 11 (4): 402-4.

51. Mayer HM, Brock M. Percutaneous diskectomy in the treatment of pediatric lumbar disk disease. Surg Neurol 1988 Apr; 29 (4): 311-4.

52. Ehni G, Schneider J. Posterior lumbar vertebral rim fracture and associated disc protrusion in adolescence. J Neurosurg 1988 Jun; 68 (6): 912-6.

53. Ross AC, Edgar MA, Swann M, Ansell BM. Scoliosis in juvenile chronic arthritis. J Bone Joint Surg Br 1987 Mar; 69 (2): 175-8.

54. Boston HC Jr, Bianco AJ Jr, Rhodes KH. Disk space infections in children. Orthop Clin North Am 1975 Oct; 6 (4): 953-64.

55. Scoles PV, Quinn TP. Intervertebral discitis in children and adolescents. Clin Orthop Relat Res 1982 Jan-Feb; 162: 31-6.

56. Hernigou PH, Nabih A, Goutallier D. Hidatidose vertébrale: complications, apport de l'imagerie moderne. Rev Rhum Mal Osteoartic 1992 Fev; 59 (2): 131-5.

Os autores declararam não possuir conflitos de interesses

\section{ENDEREÇO PARA CORRESPONDÊNCIA}

Prof. Dr. Jorge Mineiro

Tel: 210025354

E-mail: jorge.mineiro@hospitalcufdescobertas.pt

\section{ABSTRACT}

\section{BACK PAIN IN CHILDREN}

Back pain is one of the most common musculoskeletal complaints in the adult population. However, its relevance in children and adolescents is somewhat controversial. A recent article has pointed out a very high incidence of the pathology in this young age group which in fact brings this condition to the attention of the public health physicians.

In the adult population the main causes of back pain are usually related either to degenerative conditions or to other less common sources like psychological disturbances and other psychosomatic illnesses. In children the most common etiologies are definitely organic based and not related to the wear and tear of the anatomic structures or to underlying psychological pathologies.

In the presence of a child with back pain the algorithm for treatment should be based on the child's presentation, on the severity of symptoms, neurologic complications or other extra spinal signs. In the absence of these symptoms and signs, patients should be followed up adequately before initiating investigations that are often time consuming, and expensive, causing pain and distress with low probability of success.

Keywords: Back Pain; Child; Adolescent. 